\title{
Effective Elimination of Laser Interference Fringing in Fluorescence Microscopy by Spinning Azimuthal Incidence Angle
}

\author{
ALEXA L. MATTHEYSES, ${ }^{1}$ KEITH SHAW, ${ }^{1}$ AND DANIEL AXELROD ${ }^{1,2 *}$ \\ ${ }^{1}$ Biophysics Research Division, University of Michigan, Ann Arbor, Michigan 48109 \\ ${ }^{2}$ Department of Physics, University of Michigan, Ann Arbor, Michigan 48109
}

\section{KEY WORDS illumination; coherence; uniformity; dephasing}

\begin{abstract}
Laser illumination used in both conventional widefield epi-fluorescence as well as in total internal reflection fluorescence (TIRF) microscopy is subject to nonuniformities in intensity that obscure true image details. These intensity variations are interference fringes arising from coherent light scattering and diffraction at every surface in the laser light's optical path, including the lenses, mirrors, and coverslip. We present an inexpensive technique for effectively eliminating these interference fringes based upon introduction of the excitation laser beam by oblique throughthe-objective incidence coupled with rapid azimuthal rotation of the plane of incidence. Although this rotation can be accomplished in several ways, a particularly simple method applicable to a free laser beam is to use an optical wedge, spun on a motor, which diverts the beam into a hollow cone of fixed angle. A system of lenses converts this collimated beam cone into a focused spot that traces a circle at the objective's back focal plane. Consequently, a collimated beam with fixed polar angle and spinning azimuthal angle illuminates the sample. If the wedge is spun rapidly, then the different interference patterns at every particular azimuthal incidence angle average out over a single camera exposure to produce an effectively uniform field of illumination. Microsc. Res. Tech. 69:642647, 2006. @ 2006 Wiley-Liss, Inc.
\end{abstract}

\section{INTRODUCTION}

In laser-based microscopy illumination systems, interference fringes at the sample plane occur because of the coherent nature of the illumination. Every surface through which the excitation beam traverses causes some scattering and diffraction, thereby producing multiple mutually coherent sources of varying phases, which interfere with each other. The exact pattern of these interference fringes is very sensitive to the sample's position and composition, and so the pattern is difficult to reproduce and correct by a normalization procedure.

For accurate qualitative and quantitative microscopic imaging, it is desirable to suppress uneven illumination intensity. One type of approaches for achieving a uniform excitation field employs optical fibers. A multilength optical fiber bundle in which the fiber-tofiber length differences exceed the laser light's coherence length can produce an extended source at the output tip, with point-to-point relative phases that are temporally randomized and defocused on the sample (Inoue et al., 2001). This elegant system, with no moving parts, produces a speckle pattern that changes so rapidly that it appears uniform down to the nanosecond time scale. Commercially available mode scramblers and rapid flexing of optical fibers may also reduce some fringing. Another set of methods uses a free laser beam rather than fibers. For example, a finely frosted glass surface or a plastic plate (such as tissue culture dish bottom: see Kuhn and Pollard, 2005), spinning laterally to the laser beam, temporally randomizes the phase and produces a fringe pattern that fluctuates and can be averaged over the duration of a camera exposure. The approach presented here also uses a free laser beam but achieves the intensity averaging effect by rapidly spinning the direction from which the sample is illuminated. This eliminates not only fringes due to imperfections in the optical system but also fringes arising from irregularities on the sample itself and appears very suitable for use on living cell cultures.

The motion of the beam path is achieved here by rapidly rotating the focused beam in a circle around the back focal plane of the microscope at some off-axis radius. This leads to a collimated beam incident upon the sample at a nonnormal polar angle and a continual rotation in azimuthal angle. If the polar angle is greater than the critical angle for total internal reflection (TIR) at the sample (produced by a sufficiently large off-axis radius), the system can be used to produce an apparently uniform field of TIR excitation of fluorescence (TIRF: see Axelrod, 2003 for a review). If the polar angle is less than the critical angle, the result is an effectively uniform pattern of obliquely propagating epi-illumination.

Previously, this method has been set up in our lab using computer-controlled mirrors placed at the field diaphragm plane to rotate the beam around the back

\footnotetext{
*Correspondence to: Daniel Axelrod, Department of Physics, University of Michigan, Ann Arbor, MI 48109, USA. E-mail: daxelrod@umich.edu Received 7 December 2005; accepted 8 February 2006

Contract grant sponsor: NIH; Contract grant number: R01-NS38129. DOI 10.1002/jemt.20334

Published online 12 June 2006 in Wiley InterScience (www.interscience.wiley.com).
} 


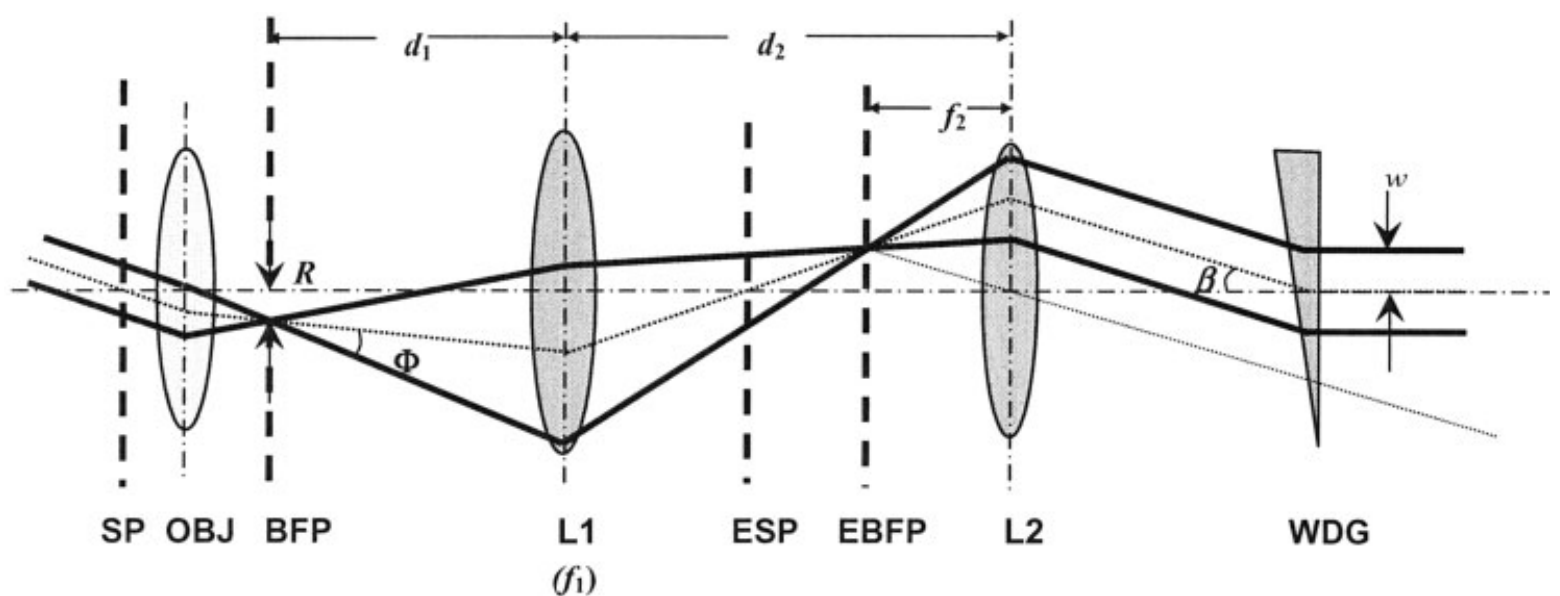

Fig. 1. Optical scheme for spinning wedge and two lenses. The collimated laser beam enters from the right and travels to the left. SP, sample plane; OBJ, objective; BFP, back focal plane of the objective; L1, lens 1; ESP, equivalent sample plane as formed by L1; EBFP, equivalent back focal plane as formed by L1; L2, lens 2; WDG, spinning wedge.

focal plane (in a system custom-built for our lab by Cambridge Research Instruments, CRI, Woburn, MA). The method presented here, using a rapidly spinning wedge of glass to rotate the beam, is not software-programmable or as versatile but is significantly less costly and produces an identical effect.

The use of a spinning wedge to suppress laser speckles by rapidly rotating azimuthal angles is not new: Bowman (1968) demonstrated it for large area, nonmicroscopic illumination. This work essentially adapts that idea to microscopy, particular fluorescence microscopy, and TIRF.

\section{MATERIALS AND METHODS Optical Scheme}

The goal of the optical scheme (Fig. 1) is to focus the laser beam at the back focal plane (BFP) at an off-axis position of radial distance $R$, the azimuthal angle of which can be rapidly changed. The fixed lens closest to the microscope, L1, forms planes equivalent to the microscope sample plane (ESP) and the objective back focal (EBFP) in free space as shown.

As the beam approaches the microscope, it first passes through a hollow shaft that holds a glass plate with planar but slightly nonparallel surfaces (a "wedge," available commercially in a variety of sizes and wedge angles from Edmund Industrial Optics, Barrington, NJ). The wedge diverts the beam into an off-axis direction without altering its collimation. The wedge is rotated by a motor so that the deflected beam traces the wall of a cone.

The deflected but still-collimated beam next passes through fixed lens L2, which focuses the light off-axis at the EBFB, at which the focused spot traces a circle as the wedge rotates. The diverging beam then crosses the optical axis at the ESP, thereby assuring that the center of the field of view is illuminated regardless of the azimuthal angle of rotation of the beam. Lens L1 subsequently focuses the beam off-axis at the actual

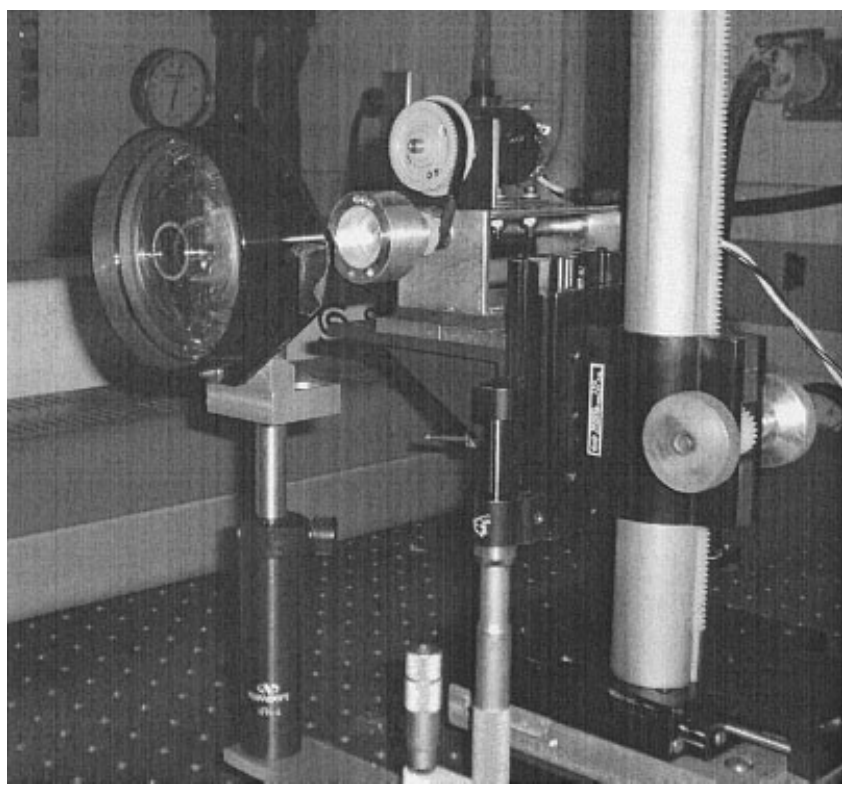

Fig. 2. Photograph of spinning wedge mount with motor and belt drive, and lens L2. The laser beam enters from the left, travels down a hollow shaft, and out through the wedge. The ring of light seen on L2 is the trace of the deflected beam as it rotates rapidly.

BFP of the objective, so that the light incident upon the sample is collimated at a fixed polar angle.

The goal is to illuminate the sample with collimated light directed at some chosen polar incidence angle (which must exceed the critical angle for TIR illumination), with a width adequate to illuminate the entire observation field. These two observable illumination features, polar angle and field width, are determined by the off-axis radius $R$ and the convergence angle $\Phi$ at the BFP, respectively. These parameters are not observed or measured directly and are different for each particular objective. Achieving the desired $(R, \Phi)$ pair can be done 

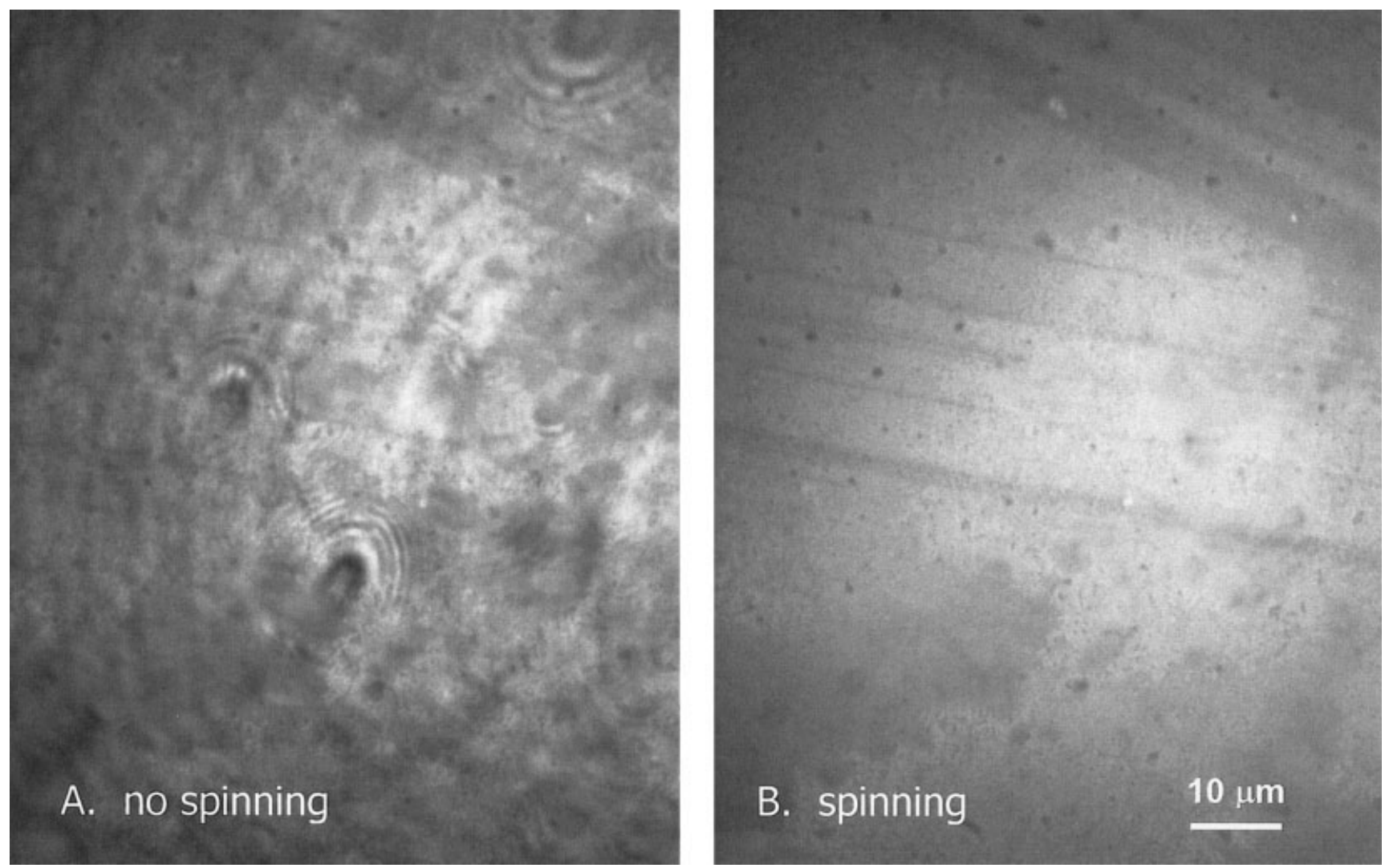

Fig. 3. TIRF images of diI adsorbed to a glass coverslip. (A) Sample illuminated with a single azimuthal incidence angle, i.e. wedge not spinning. Interference fringes are very evident. (B) Same field of view with wedge spinning. The laser interference fringes are no longer visible. The remaining nonuniformities are due to features of the sample and also the fading of illumination intensity toward the corners.

with a variety of combinations of lens positions $\left(d_{1}\right.$ and $\left.d_{2}\right)$, focal lengths $\left(f_{1}\right.$ and $\left.f_{2}\right)$, wedge deflection angle $(\beta)$, and incoming laser beam half-width $(w)$.

Simple geometrical analysis of the Figure 1 ray diagram (in the small angle approximation) leads to a rule specifying the wedge deflection angle

$$
\beta=\frac{R \Phi}{w} .
$$

The wedge deflection angle $\beta$ that is necessary for this system does not depend on the choices for $d_{1,2}$ and $f_{1,2}$, but depends only on the incoming beam width and the optics of the chosen objective. Given a free choice of the ratio $d_{1} / f_{1}$, the desired convergence angle $\Phi$ can be achieved by a proper choice of focal length $f_{2}$,

$$
f_{2}=\frac{w}{\Phi\left(\frac{d_{1}}{f_{1}}-1\right)} .
$$

Given a free choice of the distance $d_{1}$, the distance $d_{2}$ can be calculated as follows:

$$
d_{2}=f_{2}+\frac{d_{1}}{\left(\frac{d_{1}}{f_{1}}-1\right)} .
$$

The actual parameters used in our setup were approximately $\beta=6^{\circ} ; f_{1}=30 \mathrm{~cm} ; f_{2}=7 \mathrm{~cm} ; d_{1} \approx 50 \mathrm{~cm} ; d_{2} \approx$ $80 \mathrm{~cm} ; w=0.15 \mathrm{~cm}$. Two different Nikon objectives were used: a $60 \times$ NA 1.45 and a $60 \times$ NA 1.48 (prototype). Switching between them required slight relative adjustments to distances $d_{1}$ and $d_{2}$.

Another possible optical arrangement not implemented here involves a single external lens, as discussed in the Appendix.

\section{Design of Wedge Spinner}

Figure 2 shows a photograph of the wedge spinner and lens L2. The wedge (Edmund's Industrial Optics) is mounted with optical grade silicone adhesive to an aluminum head attached by Loctite 608 glue to a hollow shaft. The $6 \mathrm{~mm}$ inside diameter of the shaft provides ample clearance for the optic beam. The wedge, head, and shaft assembly is carefully balanced to compensate for the off-center center-of-mass of the wedge.

The optical wedge assembly can be rotated up to 580 rps by a $100 \mathrm{~W}$ direct current (DC) brushed motor through a 3:1 step-up belt drive consisting of Stock Drive Products timing belts and pulleys. The belt drive configuration was chosen to minimize vibration coupling to the optical table, further reduced by Lord Mounts between the motor, drive chassis, and optical table. 

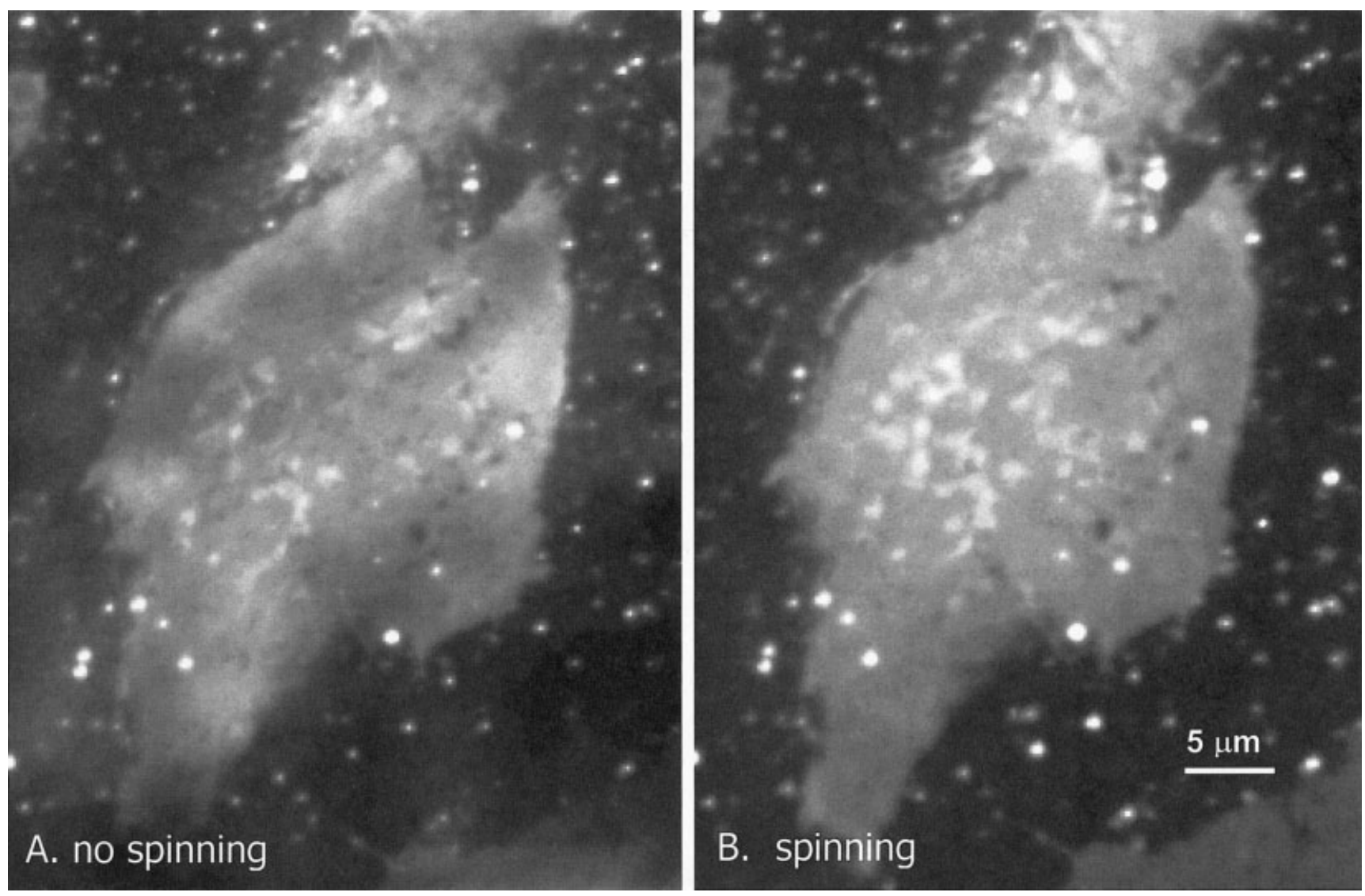

Fig. 4. TIRF images of diI labeling in the plasma membrane of a HEK cell. (A) Sample illuminated with a single azimuthal incidence angle; wedge not spinning. Interference fringes of characteristic size around 5-10 $\mu \mathrm{m}$ are evident. (B) Same field of view with wedge spinning. The interference fringes are no longer evident.

It is desirable to have enough complete rotations of the wedge so that partial rotations are not significant in a single exposure (unless the rotation period is somehow coupled to the camera exposure time). For example, with an exposure time of $0.1 \mathrm{~s}$ and a goal of 10 rotations per exposure, the rotation speed would have to be at least $100 \mathrm{rps}$.

\section{Optical Details}

The laser employed here was a water-cooled argon ion model (3 W maximum total power, Coherent Inc., Santa Clara, CA), operated at wavelength $502 \mathrm{~nm}$, and optical filters and dichroics appropriate for "rhodamine" fluorescence (Chroma Technology Corp., Rockingham, VT). The microscope was a Nikon TE2000, equipped with either a $60 \times$ NA 1.45 (for noncell experiments) or a prototype $60 \times$ NA1.48 (for cell experiments). The microscope's rear port where a mercury arc lamp housing would normally be mounted was removed, along with the associated lens optics. This is where the laser beam entered the microscope.

The detector was a Sensicam QE CCD camera $(1376 \times$ 1040 pixels, Cooke Corporation, Romulus, MI) mounted on the microscope's side port. Camera exposures were
1-2 s duration, controlled by Sensicam's Sensicontrol software.

\section{Sample Preparation}

Two types of samples were observed. One was a layer of the fluorescent carbocynanine diI- $\mathrm{C}_{18^{-}}(3)$ (diI, Molecular Probes, Eugene, OR) adsorbed to a glass coverslip and the other was diI labeled living cells on a coverslip. The noncell sample was prepared by placing a drop of saturated diI/ethanol solution on a glass coverslip and then rinsing off with distilled water a few seconds later. A drop of phosphate buffered saline (PBS) was placed on the coverslip before observation. The cell sample was prepared by adding $20 \mu \mathrm{L}$ of saturated diI/ethanol solution to $1 \mathrm{~mL}$ of PBS bathing a $25 \mathrm{~mm}$ diameter glass coverslip, on which human embryonic kidney (HEK) cells had been grown to a sparse density. After about $5 \mathrm{~min}$ of incubation at room temperature, the cells were rinsed with PBS and then observed. DiI preferentially labels the plasma membrane, at least in the first few tens of minutes before it becomes obviously internalized. 


\section{RESULTS AND DISCUSSION}

Fluorescent images of the diI-labeled glass are shown illuminated by a single incidence angle in Figure 3A (wedge in fixed position) and illuminated by a $2 \pi$ continuous range of azimuthal incidence angles in Figure 3B (wedge spinning). Note that the majority of the intensity variations present in Figure 3A are eliminated by the spinning. There are two types of variations remaining in Figure 3B. One arises from spatial variations in the illumination present at all azimuthal angles, such as the central brightness/peripheral darkness. The other arises from actual variations on the sample, such as the streaks, scratches, holes, and smears typical of diI labeling on commercial coverslips. With spinning, these sample variations that were obscured because of interference fringes become obvious.

Fluorescent images of the diI-HEK cell sample are shown in Figure 4A (wedge in fixed position) and Figure 4B (wedge spinning). Again, spinning removes artifactual intensity variations on the several micrometer spatial scale that otherwise could easily be misidentified as cellular features. Cells with significant optically dense inhomogeneities (not present here) often cast dark shadows "downstream" in the direction of the phase propagation of the incident field. With azimuthally spinning illumination, such directional shadows are much reduced in contrast and spread into halos. Other methods of speckle reduction (such as phase scrambling) that do not involve directional spinning do not have this effect.

Azimuthal rotation affects the polarization of the light at the sample. Consider the polarizations for supercritical polar angle (i.e., TIR) illumination, and the field of view mapped in "east, north, west, and south" directions. A vertically polarized incoming beam is "s-polarized" when it travels from the east on the sample plane, becoming p-polarized as rotates around to originate from the north, back to s-polarized from the west, and p-polarized again from the south. This polarization-rotating effect may seem to be a problem for applications where it is desirable to maintain some fixed polarization of the excitation light, but speckle reduction is still desirable. One such application is polarized fluorescence resonance energy transfer (PFRET, see Mattheyses et al., 2004) in which illumination uniformity and distinct polarizations are simultaneously required. However, we have observed that interference fringe elimination does not require a full $360^{\circ}$ of azimuthal rotation; often only about $30^{\circ}$ is adequate. Over that small range, polarization mixing is not severe.

The spinning wedge system described here is an inexpensive method of producing azimuthal rotation. Azimuthal rotation has been used in our lab to study secretion from chromaffin cells (Allersma et al., 2006) using a different system custom designed by Cambridge Research Instruments (CRI, Woburn, MA) that employs two small mirrors mounted on a pair of computer-controlled rotational actuators located near the field diaphragm plane (i.e., equivalent sample plane) just outside the microscope. The CRI system is programmable, easier to align, and creates less vibration, but the wedge system described here is much cheaper and illuminates a significantly larger portion of the field of view. A system that potentially could eliminate all moving parts and thereby all vibrations might consist of two orthogonally oriented acousto-optic modulators (AOM) used in place of the wedge. These AOM's would be driven by microwave carriers that modulate in frequency sinusoidally, with the two AOM's driven out of phase by $90^{\circ}$. That protocol would produce a circular path of collimated light identical to that produced by the spinning wedge.

The spinning azimuthal angle system works for both super- and subcritical polar angles. Even at a polar angle of zero, the technique should blur out interference fringes that arise from imperfections or dust on surfaces in the optical path. But at nonzero polar angles, the spinning azimuthal angle system provides the additional and unique advantage of blurring out interference nonuniformities and shadows created by the sample itself.

\section{APPENDIX Single Lens Optical Scheme}

Rather than incorporating two lenses L1 and L2, the system can be arranged with just one lens external to the objective (see Fig. A1). This arrangement is simpler but with a countervailing loss of flexibility in choice of focal length and position.

In Figure A1(A), the centerline of the light path crosses the optical axis at the sample plane (SP) and also crosses to the right of lens L, at an ESP created by lens L. The wedge WDG must be positioned at this ESP so that the illuminated region on the sample remains fixed as the wedge rotates. This is why the BFP-L distance and the L-WDG/ESP distance both equals to the focal length $f$ of lens $L$.

Given the required values of $R$ and $\Phi$ determined by the objective (as discussed in the main text), we can determine $f$. Again assuming small angles

$$
\begin{aligned}
& f=R / \beta \\
& f=w / \Phi
\end{aligned}
$$

Both of these requirements must be satisfied. Reasonable rough estimates of $\Phi \approx 0.1 \mathrm{rad}$ and $w \approx 0.15$ $\mathrm{cm}$ lead to $f \approx 1.5 \mathrm{~cm}$. Since $R$ needs to be roughly 0.3 $\mathrm{cm}$ to produce TIR through a typical high aperture objective, we must have $\beta \approx 0.2 \operatorname{rad}\left(11^{\circ}\right)$.

This short $f$ may require that both the lens $\mathrm{L}$ and the spinning wedge be installed inside the microscope just upbeam from the dichroic mirror. In principle, $f$ can be made larger (and $\beta$ smaller) proportionately with an expanded beam width w. Another approach that allows $\mathrm{L}$ to back away from the BFP is to place a diverging lens on-axis immediately to the right (upbeam) from the spinning wedge (Fig. A1(B)). This will not change the requirements given by Eqs. (A1) and (A2), but it will allow for a distance $d$ from $L$ to the BFP to be set larger than $f$, giving more working room. Of course, this arrangement is not strictly "one lens," but the divergent lens' position is fixed near the wedge and can be designed into the right (entrance) surface as a concavity in the wedge itself. 

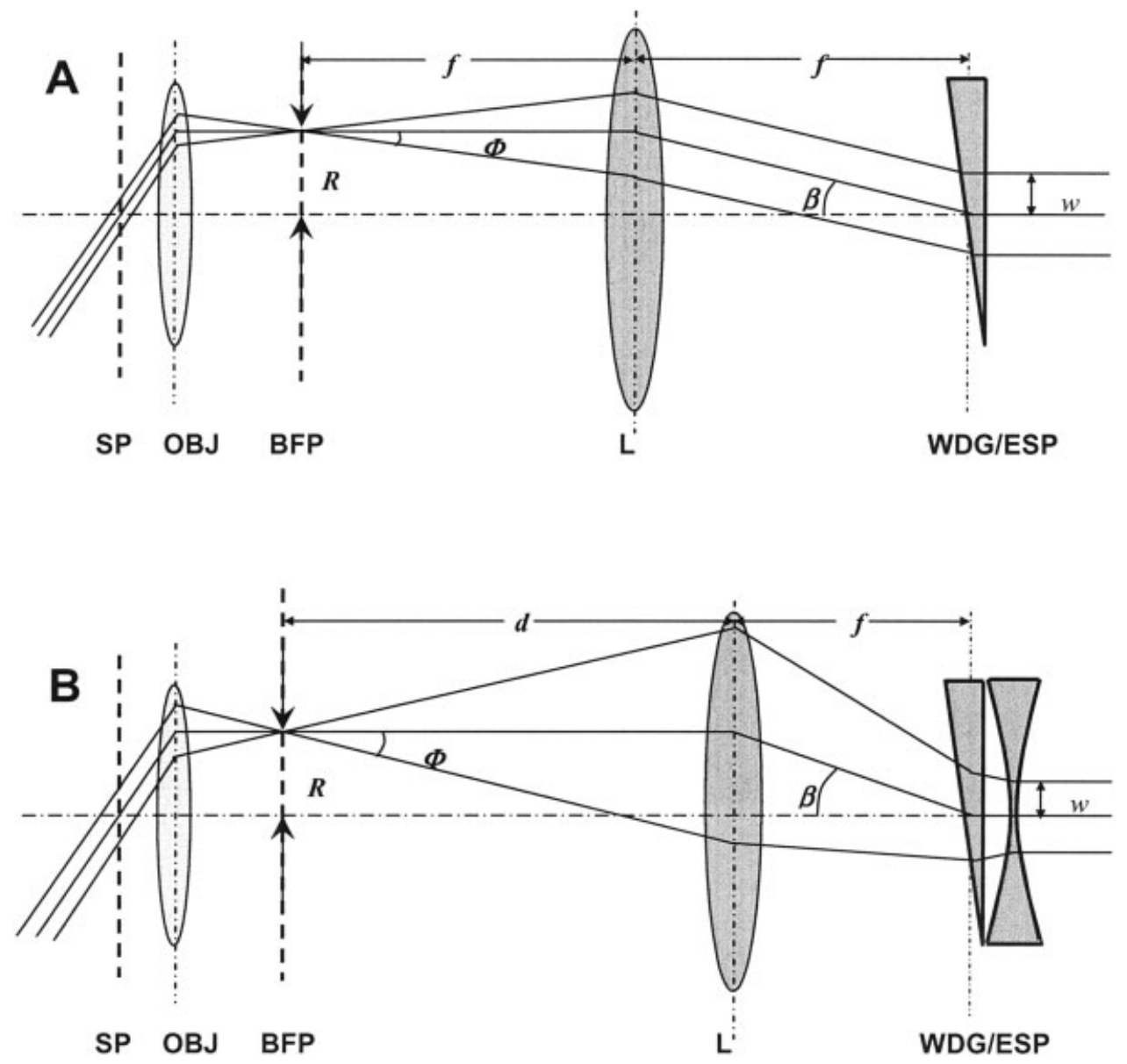

Fig. A1. Optical scheme for spinning wedge and one lens. SP, sample plane; OBJ, objective; BFP, back focal plane of the objective; L, lens; ESP, equivalent sample plane as formed by L; WDG, spinning wedge. (A) Collimated laser light enters the system from the right.

(B) Divergent light enters the system from the right. The divergence is produced by a concave lens immediately up-beam from the wedge. (Alternatively, a custom wedge with a concavity in its entrance surface, not shown, could replace the divergent lens/wedge combination.)

\section{ACKNOWLEDGMENTS}

The authors thank Nikon Instruments for the loan of the TE2000 inverted microscope and associated objectives. They also thank Cambridge Research Instruments, Inc. and in particular Drs. Richard Levenson and Paul Cronin for many useful discussions about azimuthal angle rotation.

\section{REFERENCES}

Allersma MW, Bittner MA, Axelrod D, Holz RW. 2006. Motion matters: Secretory granule motion adjacent to the plasma membrane and exocytosis. Molecular Biology of the Cell 17: 2724-2738.

Axelrod D. 2003. Total internal reflection fluorescence microscopy in cell biology. Methods Enzymol 361:1-33.

Bowman MJ. 1968. Two new methods of improving optical image quality. Appl Optics 7:2280-2284.

Inoue S, Knudson RA, Goda M, Suzuki K, Nagano C, Okada N, Takahashi H, Ichie K, Iida M, Yamanak K. 2001. Centrifuge polarizing microscope. I. Rationale, design, and instrument performance. J Microsc 201:341-356.

Kuhn JR, Pollard D. 2005. Real-time measurements of actin filament polymerization by total internal reflection fluorescence microscopy. Biophys J 88:1387-1402.

Mattheyses AL, Hoppe AD, Axelrod D. 2004. Polarized fluorescence resonance energy transfer microscopy. Biophys J 87:2787-2797. 